\title{
Adsorption of $\mathbf{N}, \mathbf{N}$-dimethylamine from aqueous solutions by a metal organic framework, MOF-235
}

\section{( 遇 Article in press ? )}

- Beltrán-Suito, R. ${ }^{\mathrm{a}, \mathrm{b}}$,

- Pinedo-Flores, A. ${ }^{\mathrm{a}}$,

- Bravo-Hualpa, F. ${ }^{a}$,

- Ramos-Muñoz, J.a,c,

- Sun-Kou, M.D.R. Email Author

- View Correspondence (jump link)

- ${ }^{a}$ Department of Sciences, Section Chemistry, Pontifical Catholic University of Peru, San Miguel, Peru

- ${ }^{b}$ Department of Chemistry, Metalorganic Chemistry and Inorganic Materials, Technical University of Berlin, Berlin, Germany

- 'Department of Sciences, Peruvian University of Applied Sciences, Monterrico, Santiago de Surco, Peru

\section{Abstract View references (22)}

Water-resistant MOF-235 was used to adsorb N,N-dimethylamine (DMA) from aqueous solutions. It was synthesized from terephthalic acid and $\mathrm{FeCl}_{3} \cdot 6 \mathrm{H}_{2} \mathrm{O}$ under air-free conditions and characterized by its crystalline structure, functional groups and temperature resistance. The kinetic data results were best adjusted to the pseudo-second order model $\left(\mathrm{R}^{2}>0.963\right)$. The best-fit isotherm, Langmuir model, suggested the adsorption of DMA is localized on homogenously distributed active sites on the surface. This fit was confirmed by the value of $\beta=1$ on the Redlich-Peterson model. Our study suggests that the manipulation of novel materials such as MOF-235 promises new avenues for water treatment solutions. Schematic structure of MOF-235 and its application as adsorbent. () 2018, () 2018 Taylor \& Francis.

\section{Reaxys Database Information}

(1) View Compounds

\section{Author keywords}

adsorptiondimethylamineMOFMOF-235water treatment

\section{Indexed keywords}


Engineering AdsorptionChlorine compoundsCrystalline materialsIron controlled terms: compoundsMetalorganic frameworksOrganometallicsSolutions

Engineering Crystalline structureDimethylaminesMetal organic frameworkPseudouncontrolled second order modelRedlich-Peterson modelsSchematic terms structuresTemperature resistancesTerephthalic acids

\section{Engineering}

main heading: Water treatment

- ISSN: 01932691

- Source Type: Journal

- Original language: English

- DOI: $10.1080 / 01932691.2018 .1489275$

- Document Type: Article in Press

- Publisher: Taylor and Francis Inc. 